\title{
Observations en Loire-Atlantique (France) de Stenoria analis (ScHAUM) (Coleoptera, Meloidae), cleptoparasite de Colletes hederae SCHMIDT \& WESTRICH (Hymenoptera, Colletidae)
}

\section{Gilles MAHE *}

\begin{abstract}
In September 2007 the author observed in Loire-Atlantique (France) Stenoria analis ScHAUM during the mating, the egg-laying, the hatching of the triungulins (first instar larvae of the blister beetle). Pictures taken on the site of observations show how the triungulins cling to the males of Colletes hederae SCHMIDT \& WESTRICH to reach the nest of their host bees where the larvae will complete their life cycle.
\end{abstract}

Résumé. En septembre 2007 l'auteur a observé en Loire-Atlantique (France) Stenoria analis ScHAUM lors de l'accouplement, la ponte et la naissance des triongulins (premier stade larvaire du méloïdé). Des photos prises sur le site montrent comment les triongulins s'agrippent aux mâles de Colletes hederae SCHMIDT \& WESTRICH afin de rejoindre le nid de leurs abeilles hôtes où les larves pourront poursuivre leur cycle de développement.

Mots-clés. Stenoria analis, Meloidae, triongulin, Colletes hederae.

\section{Première rencontre fortuite}

Le 16 septembre 2006, je suis à Assérac (LoireAtlantique, France, WGS84 $47,417^{\circ} \mathrm{N} 2,434^{\circ} \mathrm{W}$ ) sur un cordon sablonneux en bordure de l'étier de Pont d'Arm pour tenter de photographier des abeilles du genre Epeolus, espèces cleptoparasites de Colletes hederae SCHMIDT \& WESTRICH. Soudain je me retrouve couvert de milliers de minuscules bêtes, si petites qu'il m'est impossible de distinguer leurs formes à l'œil nu. Les animalcules se groupent en petits amas sur mes chaussures, mon jean et mon T-shirt. Pour ma femme c'en est trop, il faut rentrer immédiatement. Je sauve in extremis quelques spécimens de la machine à laver pour les observer à la loupe binoculaire.

Le même jour sur le forum Apoidea-Gallica, Eric Dufrêne signale qu'il a capturé des mâles de C. hederae portant de nombreux "parasites" autour des mandibules. Camille Thirion répond qu'il peut s'agir de triongulins, des larves de méloïdés qui accomplissent leur cycle dans les nids d'abeilles solitaires. La semaine suivante, toujours sur le forum Apoidea-Gallica, Alain Livory indique qu'il pourrait s'agir de Stenoria analis, espèce qui, d'après ce qu'il a observé dans la Manche, parasite les Colletes du lierre (Livory 1998 \& 2000). C'est ainsi que j'apprends que les animalcules qui m'ont assailli sont très probablement des triongulins de Stenoria analis SCHAUM (voir aussi Vereecken et al. 2006).

\section{Cycle général de développement des Meloidae}

Pour parvenir à l'état adulte les larves de Meloïdés doivent accomplir plusieurs métamorphoses. Le

* Gilles Mahé, Rue de la matte 18, F-44600 Saint-Nazaire, France. triongulin qui est parvenu jusque dans le nid de l'abeille commence par dévorer l'œuf de son hôte puis se mue en une larve secondaire qui consommera toutes les provisions de la cellule. Cette larve secondaire se transformera en pseudonymphe d'où émergera une troisième larve qui à son tour se transformera en une vraie nymphe d'où éclora l'adulte.

La plupart des articles sur le comportement des Melödés font référence aux observations de J.H. Fabre (1882) sur Meloe proscarabaeus L., ou sur Sitaris muralis FORSTER (= Sitaris humeralis FIGURIER). Dans le cas de $M$. proscarabaeus, les triongulins postés sur les fleurs s'agripperaient aux abeilles au moment du butinage. Dans le cas de S. muralis, les œufs seraient pondus directement à l'entrée des nids des anthophores où les triongulins attendent patiemment l'émergence des abeilles pour s'y agripper. Hafernik \& Saul-Gershenz (2000) rapportent qu'un meloïdé américain, Meloe franciscanus VAN DYKE, utilise un autre subterfuge pour attirer des mâles d'Apidae du genre Habropoda : les triongulins se regroupent en amas sur des tiges végétales imitant grossièrement la forme d'une abeille et produisant une substance volatile mimétique des phéromones sexuelles des femelles d'Habropoda. Lorsqu'un mâle tente de s'accoupler avec ce leurre, les triongulins s'accrochent à ses poils et se font transporter jusqu'à ce qu'il s'accouple avec une femelle. Les triongulins passent alors sur le corps de la femelle qui les transportera jusqu'à son nid. On trouve peu d'informations sur $S$. analis et en particulier sur le procédé qui permet aux triongulins de rejoindre le nid de l'abeille hôte. D'après Mayet (1875) S. analis (= Sitaris colletis V.M.) pondrait dans les galeries des abeilles. Villemant (2001) décrit la ponte de $S$. analis sur les arbres et arbrisseaux, ainsi que la naissance des 


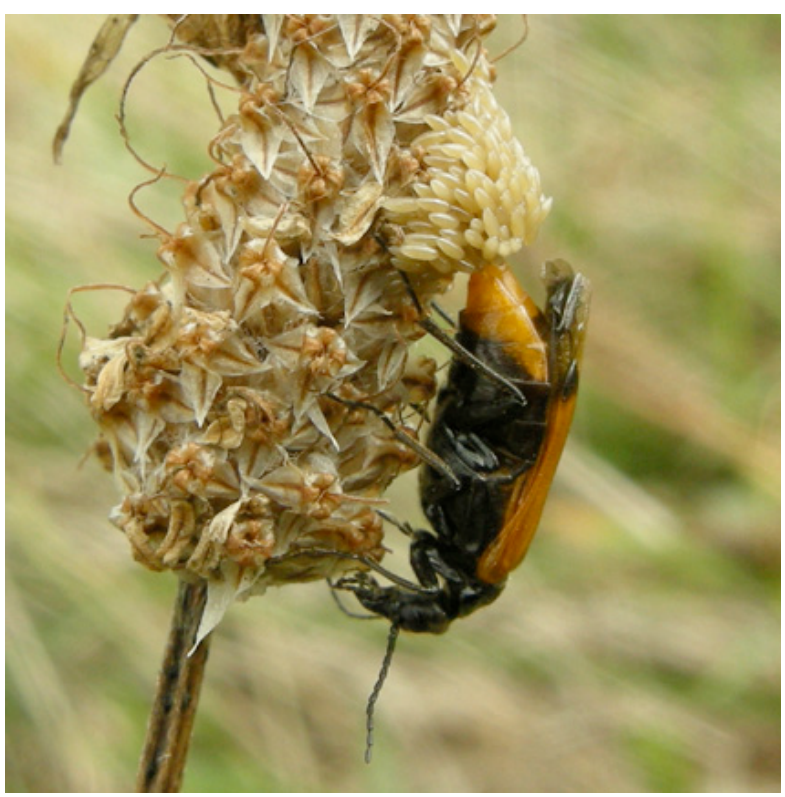

Figure 1. Stenoria analis (SCHAUM) (Coleoptera, Meloidae) sur Plantago lanceolata L. (Plantaginaceae) (Photo G. Mahé)

triongulins et la formation de masse grouillante en forme de gouttes. Les triongulins tomberaient alors au sol pour se disperser et s'accrocher aux abeilles qui les approcheraient. Ce que nous avons observé en 2007 à Assérac laisse à penser que le stratagème du méloïdé américain est aussi à l'œuvre chez $S$. analis (Vereecken \& Mahé, 2007). Rien de tel n'avait encore été décrit en Europe.

\section{Observations en 2007 à Assérac}

Le 24 août 2007, Aurélia Lachaud m’accompagne pour me montrer une micro-falaise en bordure du traict de Mesquer (Loire-Atlantique, France, WGS84 $47,413^{\circ} \mathrm{N} 2,442^{\circ} \mathrm{W}$ ) où elle a observé une belle population d'anthophores au printemps. En arrivant sur les lieux nous avons la surprise de voir sur la falaise quelques couples de $S$. muralis mais aussi des dizaines de S. analis dont plusieurs en accouplement. Nous décidons alors d'aller voir ce qui se passe à Assérac. Là, nous trouvons des dizaines de femelles de $S$. analis pondant sur tout type de support : des espèces végétales telles que Daucus carota, Plantago lanceolata, Dactylis glomerata (Figures 1 à 3) mais aussi sur le fil barbelé (Figure 4). Nous sommes retournés sur le site au moins deux fois par semaine pendant environ un mois. Nous avons observé des accouplements (Figure 5) et des pontes du méloïdé jusqu'à mi-septembre. Les premiers mâles de C. hederae ont été observés le 5 septembre et les premiers amas de triongulins (Figure 6) le 8 septembre. Le 15 septembre je note que les mâles de $C$. hederae qui patrouillent sont manifestement intrigués, et font souvent du sur place à quelques centimètres des petits paquets de triongulins fixés aux végétaux. Mais en général, ils passent leur chemin ! Sauf une fois où j'observe enfin un mâle se jetant sur les triongulins. Je me demande si l'événement est accidentel. Le contact a été bref. Il est possible que très rapidement le mâle s'est rendu compte de la supercherie. La prise de photo

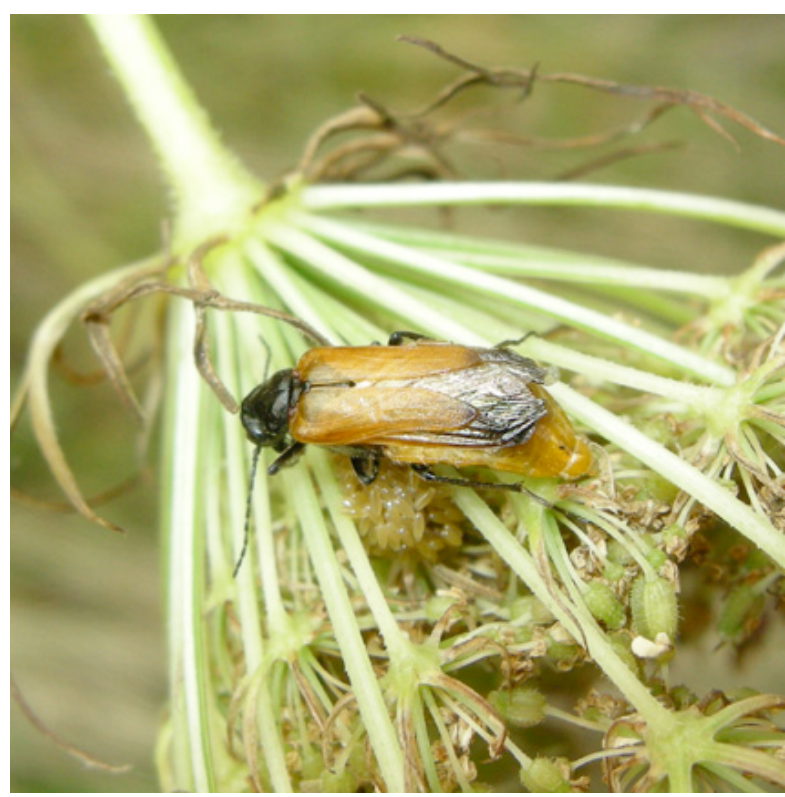

Figure 2. Stenoria analis (SCHAUM) (Coleoptera, Meloidae) sur Daucus carota L. (Apiaceae) (Photo G. Mahé)

s'avérait difficile. Pour y parvenir, le 22 septembre je prélève une tige de plantain portant un amas de triongulins que je déplace à une trentaine de mètres dans un massif de lierre. La tige de plantain est plantée dans une feuille de lierre. Et là ce fût extraordinaire. Les triongulins ont subi immédiatement les assauts répétés de plusieurs mâles de C. hederae. En quelques secondes, avant même que j'ai eu le temps de faire la mise au point de mon appareil photo, il n'y avait plus de triongulins sur le plantain. J'ai dû répéter l'opération plusieurs fois avant de trouver la technique pour prendre des photos (Figures 7 et 8). Des triongulins se fixaient presque systématiquement au niveau des mandibules des mâles de Colletes (Figures 9 et 10). Certains mâles chargés de triongulins revenaient à la charge (Figure 11). D'autres fois des mâles repartaient avec tellement de triongulins (Figures 12 et 13) qu'ils devenaient la cible d'autres mâles.

J'ai rapporté à la maison quelques pontes de triongulins pour observer leur développement. Quelques jours après leur naissance, il est curieux de les voir produire des fils de soie auxquels ils se suspendent en amas globuleux (Figure 14). Je n'ai cependant pas eu l'occasion de voir d'amas suspendus sur le terrain. Les amas de triongulins restent en général collés aux végétaux. Il serait intéressant de savoir si ce stade en "amas suspendu" a pour but d'attirer encore plus l'attention des mâles de Colletes, ou s'il s'agit d'une étape intermédiaire avant la chute au sol des triongulins dans l'espoir d'une rencontre certes plus hasardeuse mais néanmoins possible avec les Colletes dont les nids se trouvent au pied des végétaux.

\section{En conclusion}

Le phénomène observé ressemble beaucoup à celui décrit par Hafernik \& Saul-Gershenz (2000) concernant le méloïdé américain, $M$. franciscanus. Des 

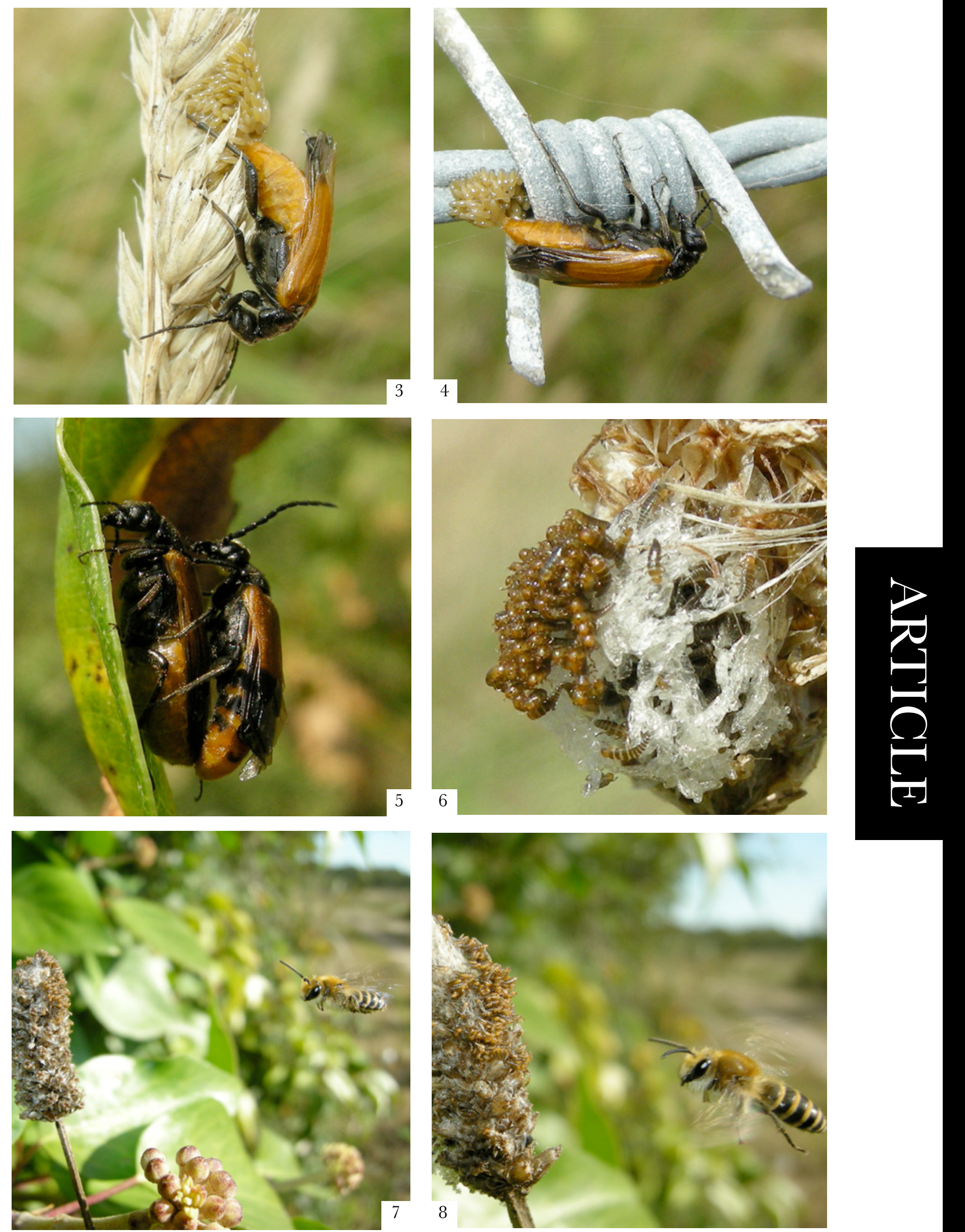

Figures 3 à 8 . Histoire naturelle de l'interaction entre Stenoria analis (SCHAUM) (Coleoptera, Meloidae) et Colletes hederae SCHMIDT \& WESTRICH (Hymenoptera, Colletidae) (Photos G. Mahé) 

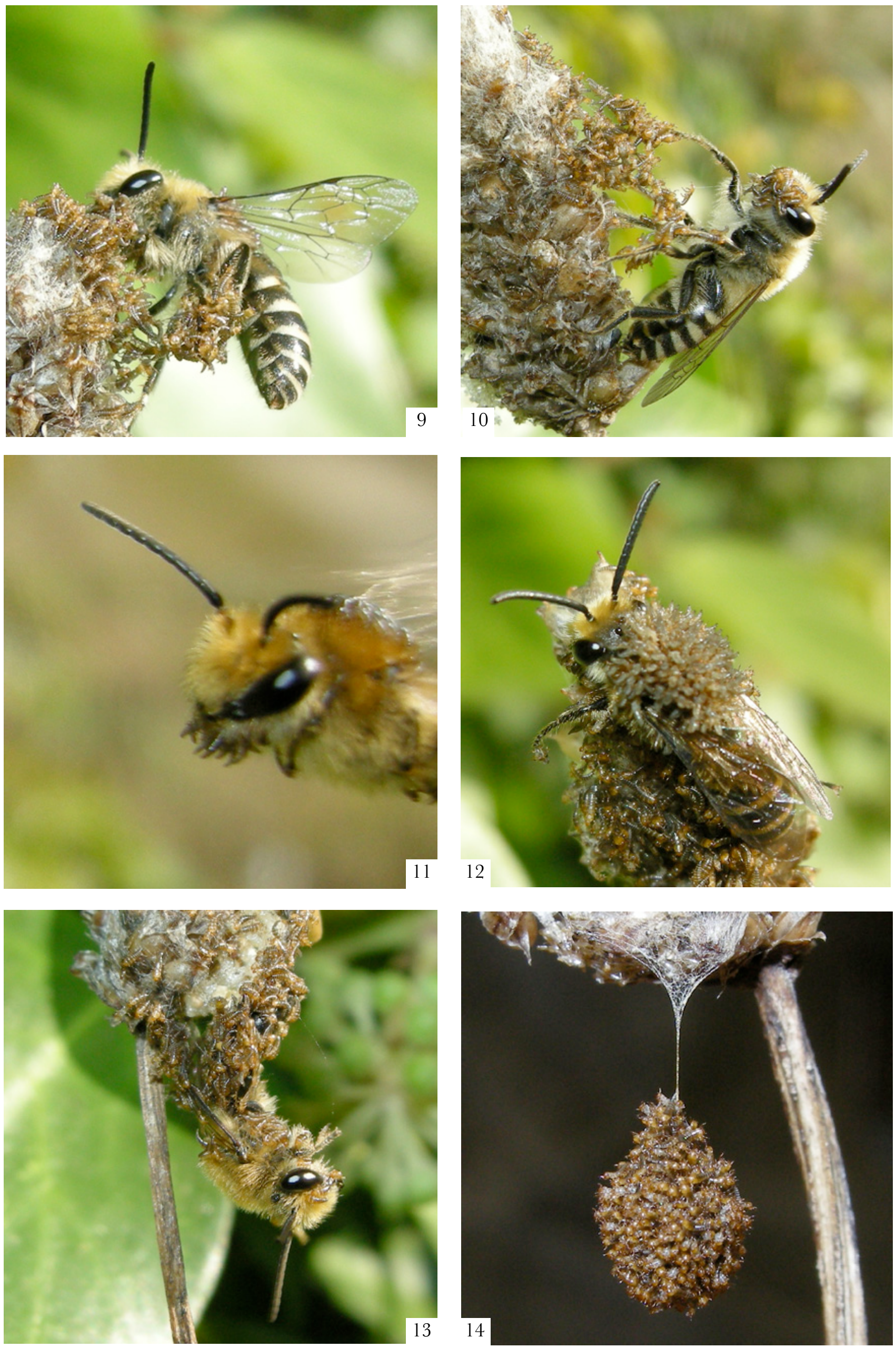

Figures 9 à 14. Histoire naturelle de l'interaction entre Stenoria analis (SCHAUM) (Coleoptera, Meloidae) et Colletes hederae SCHMIDT \& WESTRICH (Hymenoptera, Colletidae) (Photos G. Mahé) 
analyses chimiques devraient permettre d'étayer l'hypothèse de l'attractivité des amas de triongulins par des substances volatiles mimétiques des phéromones sexuelles des femelles de $C$. hederae.

\section{Remerciements}

Je remercie vivement Aurélia Lachaud (Guérande, France) qui m'a fait découvrir les sites de Mesquer et d'Assérac que nous avons régulièrement visités ensemble de fin septembre à fin octobre 2007. Je remercie tout particulièrement Nicolas Vereecken (Université Libre de Bruxelles, Belgique) qui a éveillé ma curiosité, m'a fait connaître le cas du Meloe américain et m'a incité vivement à retourner sur le terrain pour observer, prendre des photos et faire des prélèvements. Merci également à Denis Michez (Université de MonsHainaut, Belgique) d'avoir bien volontiers accepté de relire et corriger cet article. Ces observations ont fait l'objet d'un article publié dans les Annales de la Société Entomomlogique de France (voir Vereecken \& Mahé 2007).

\section{Références bibliographiques}

Fabre J-H, 1882. Nouveaux souvenirs entomologiques : études sur l'instinct et les moeurs des insectes. [2]. Paris, Ch. Delagrave, 389p. Hafernik J \& Saul-Gershenz LS, 2000. Beetle larvae cooperate to mimic bees. Nature 405: 35-36.

Livory A, 1998. Faune chausiaise : une surprise de taille. L'Argiope 22 : $13-18$

Livory A, 2000. "L'abeille de Chausey" enfin démasquée! L'Argiope 27: $47-54$.

Saul-Gershenz LS \& Millar JG, 2006. Phoretic nest parasites use sexual deception to obtain transport to their host's nest. Proceedings of the National Academy of Sciences of the USA 103(38): 14039-14044.

Vereecken NJ, Toffin E \& Michez D, 2006. Observations relatives à la biologie et à la nidification d'abeilles psammophiles d'intérêt en Wallonie. 2. Observations estivales et automnales. Parcs et Réserves 61(4): 12-20.

Vereecken NJ \& Mahé G, 2007. Larval aggregations of the blister beetle Stenoria analis (SCHAUM) (Coleoptera: Meloidae) sexually deceive patrolling males of their host, the solitary bee Colletes hederae SCHMIDT \& WESTRICH (Hymenoptera: Colletidae). Annales de la Société Entomologique de France 43(4) : 493-496.

Villemant $\mathbf{C}$, 2001. Les coléoptères méloïdés cleptoparasites des nids d'abeilles solitaires. Insectes 121: 7-10. 